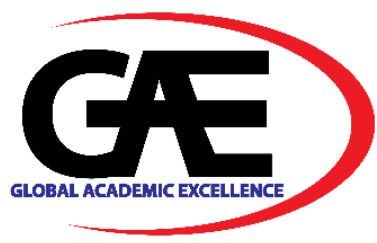

\title{
ESTIMATION OF GPS DISPERSIVE AND NON-DISPERSIVE NETWORK CORRECTION FOR ISKANDARnet NETWORK- BASED REAL-TIME KINEMATIC (N-RTK) POSITIONING SYSTEM
}

\author{
Nazrin Afiq Abdul Rahman ${ }^{1 *}$, Tajul Ariffin Musa ${ }^{1,2}$, Wan Anom Wan Aris ${ }^{1}$, Abdullah Hisam Omar ${ }^{1}$ \\ 1 Geomatic Innovation Research Group (UTM-GnG), Faculty of Built Environment and Surveying, Universiti \\ Teknologi Malaysia, Malaysia \\ Email: nazrin.afiq@gmail.com; wananom@utm.my; abdullahhisham@utm.my \\ 2 Centre of Tropical Geoengineering, Universiti Teknologi Malaysia, Malaysia \\ Email: tajulariffin@utm.my \\ * Corresponding Author
}

\section{Article Info:}

Article history:

Received date: 01.10 .2021

Revised date: 01.11.2021

Accepted date: 20.11.2021

Published date: 01.12.2021

\section{To cite this document:}

Rahman, N. A. A., Musa, T. A., Aris, W. A. W. \& Omar, A. H. (2021). Estimation of GPS Dispersive and Non-Dispersive Network Correction for ISKANDARnet Network-Based Real-Time Kinematic (N-RTK) Positioning System. Journal of Information System and Technology Management, 6 (24), 312-325.

DOI: $10.35631 /$ JISTM.624029

This work is licensed under CC BY 4.0

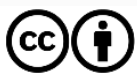

Abstract:

The concept of N-RTK positioning has been extensively developed in order to better model the distance-dependent errors of GPS carrier-phase measurements. These errors can be separated into a frequency-dependent or dispersive component (i.e., the ionospheric delay) and a non-dispersive component (i.e., the tropospheric delay and orbit biases) to express the network correction in order to attain better modelling of GPS distance dependent errors. However, the N-RTK performance may degrades due to severe atmospheric irregularities that would seriously affect the modelling of the GPS distancedependent errors, thus affecting the quality of network correction generation. The development of integrity monitoring for network correction would be great idea to identify the quality and reliability of network correction data dissemination. Therefore, this paper aims to estimates the trend of GPS dispersive and non-dispersive network correction to supports future development of integrity monitoring for network correction of ISKANDARnet N-RTK positioning system. The first part of this paper is to extract the GPS dispersive and non-dispersive network residual components. This part includes the double-differencing technique, ambiguity resolution and carrier-phased linear combination in the process. The LIM then are applied for user network coefficient value computation purpose in the second part. Finally, the GPS dispersive and non-dispersive network correction can be generated with GF and IF network correction algorithm respectively. The trend of GPS dispersive and non-dispersive network correction is expected to aid the estimation and realization of threshold limit value for development of integrity monitoring for network correction of ISKANDARnet N-RTK positioning system. 
Keywords:

N-RTK, Network Residual, Network Coefficient Value, Network Correction, Trend.

\section{Introduction}

The permanent Global Positioning System (GPS) station or Continuously Operating Reference Station (CORS) network of regional and local scales have been established in many placed around the worlds. The CORS has become a backbone infrastructure in order to improve the efficiency in range of application that requires high positioning accuracies at any time. The CORS however not only being used as a single-based reference station but also as a networkbased reference station which are primarily essential for the Network-Based Real-Time Kinematic (N-RTK) positioning technique.

According to Shariff (2009), the N-RTK technique can be defined as a combination and interpolation of real-time carrier phased-based positioning from at least three CORS. The NRTK technique accounts the distance-dependent errors (i.e., atmospheric delays and orbital errors) specifically to the rover by an interpolation method which are generally based on the calculated network coefficient and estimated network residual vector within the area in term of network correction. It is necessity to model this GPS distance-dependent errors into a separated frequency-dependent or dispersive components and non-dispersive component in order to attain better quality of network correction (Musa, 2007). The carrier-phased Ionosphere-Free (IF) and Geometry-Free (GF) linear combination method can be used in this context to extract these dispersive and non-dispersive residuals components respectively.

The quality control and integrity monitoring are the most important aspect in order to examines the quality and integrity issue of real-time CORS network (Rubinov et al, 2011). A variety of quality control and integrity monitoring concept has been developed over past few years such as to monitor real-time GPS/GNSS data quality by Lim et al (2008), to assist fast identification of multiple satellite faults in the context of multi constellation by Wang et al (2009) and by using relative quality threshold by Rubinov et al (2011). Future development of integrity monitoring system for network correction of ISKANDAR CORS Network (ISKANDARnet) N-RTK positioning system is based on quality indicator or threshold limit value for network correction. In this case, the threshold limits value will be act as perturbation monitoring sensor for ISKANDARnet network correction dissemination. The new development integrity monitoring system for ISKANDARnet N-RTK is expected to improve the reliability of the network correction in term of network correction data quality for the N-RTK users. The integrity monitoring system of ISKANDARnet N-RTK positioning system will noticed either user or master the condition of network correction compared to threshold limit value.

\section{Methodology}

This paper focus on the estimation of GPS dispersive and non-dispersive network correction. There are three steps include in this process which are first (1) the estimation of GPS dispersive and non-dispersive network residual components, second (2) is the computation of network coefficient value, and third (3) is the generation of GPS dispersive and non-dispersive network correction which are based on step 1 and step 2. Figure 1 illustrate the procedure to estimates the GPS dispersive and non-dispersive network correction in this paper. 


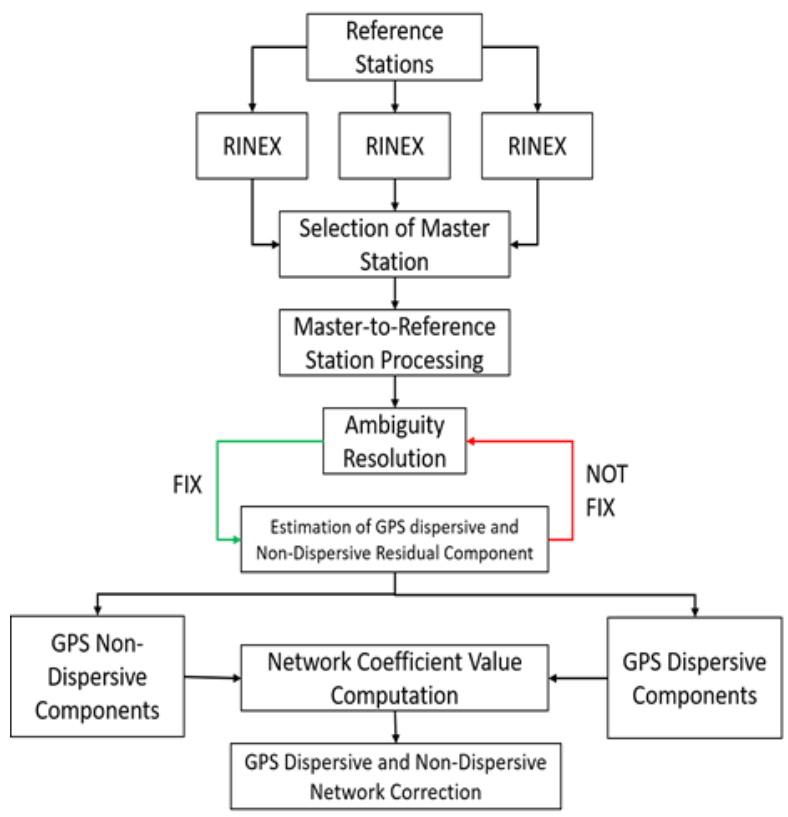

Figure 1: Procedure to Estimate GPS Dispersive and Non-Dispersive Network Correction.

To deliver such quality of GPS dispersive and non-dispersive network residual components, the geodetic GNSS software namely Bernese GNSS software is utilized. The network coefficient value and network correction are computed manually based on applied N-RTK interpolation method and GF and IF network correction algorithm respectively which will be discussed in detail.

\section{Estimation of GPS Dispersive and Non-Dispersive Network Residual Components}

There are several popular carrier-phased linear combination methods of L1 and L2 GPS/GNSS observation that has been used to eliminated certain of GPS errors according to its purpose such as Wide-Lane (WL), Narrow-Lane (NL), Ionosphere-Free (IF) and Geometry-Free (GF) (Blewitt, 1989; Nykiel \& Figurski, 2016; Zhang et al., 2019;). Each of this linear combination method has common characteristic which are for dual-frequency observables.

According to Krypiak-Gregorczyk \& Wielgosz, 2018, the multi-frequency GNSS signal and its GF linear combination can be used to model and studied the GPS dispersive component or ionospheric delay. However, the reliable modelling of ionosphere delay or GPS dispersive component is one of the most challenging aspects for precise GNSS positioning (Leick et al. 2015; Wielgosz 2011) and GNSS-based geodetic and geodynamic studies (Bosy, 2005).

In this paper, the GF and IF carrier-phased linear combination is utilized to extract separately GPS dispersive and non-dispersive network residual components. The GPS dispersive and nondispersive network residual components are extracted for two-years of GPS observation period with every 30 second epoch. To extract the GPS dispersive and non-dispersive network residual components, three reference station of ISKANDARnet has been designed, the maximum baseline length is approximately around 61 kilometers $(\mathrm{km})$. Figure 2 shows the design network of three reference station of ISKANDARnet. 


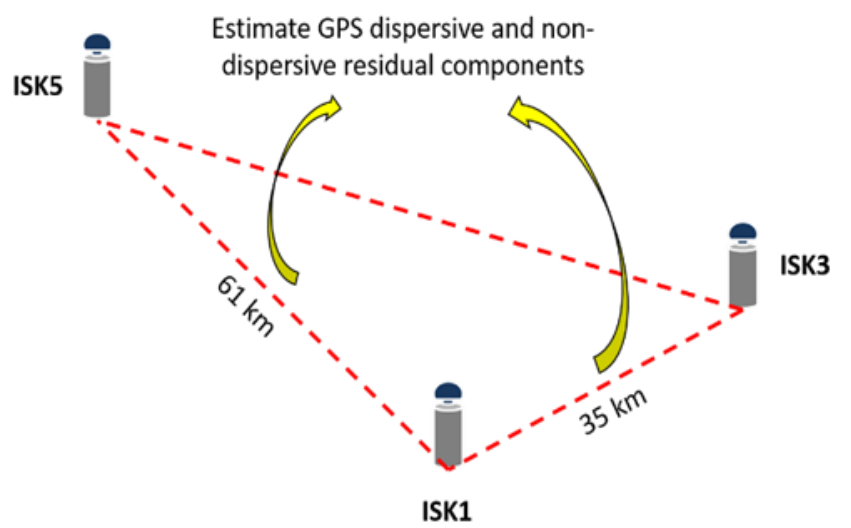

\section{Figure 2: Design Network for ISKANDARnet1 (ISK1), ISKANDARnet3 (ISK3) and ISKANDARnet5 (ISK5) of ISKANDARnet.}

To improve the quality of GPS non-dispersive network residual components, the precise ephemeris and ionosphere model are applied to minimize the effect of orbital errors (Musa, 2007) and to eliminate the higher-order ionospheric (HOI) delay (Dach et al., 2015) in the measurement respectively during the processing. In order to estimates true GPS dispersive and non-dispersive network residual components, the ambiguity for the entire network is obligatory to be resolved.

To assist the entire network ambiguity resolution, this process includes the used of carrierphased double-differenced technique, dual-frequency receiver for redundancy or linear combination, precisely known coordinate for each reference station, precise ephemeris and CORS station which are expected to have low multipath effect and clear sky environment and lastly adequate inter-station distance. Depending on the baseline length between the stations given, the network ambiguities resolution is expected to be sufficient enough to distribute the quality of GPS dispersive and non-dispersive network residual components.

\section{N-RTK Interpolation Method}

Over the past few years, the use of GPS reference station network or N-RTK to mitigates the GPS distance-dependent errors had shown great promise. In order to account these GPS distance-dependent errors, several N-RTK interpolation method had been developed and introduced such as Linear Combination Model (LCM), Linear Interpolation Method (LIM) Distance-Based Linear Interpolation Method (DIM), Lower-Order Surface Model (LSM) and Least-Squares Collocation Method (LSC).

Dai et al., 2003 shows the comparison between each of these N-RTK interpolation method in detail as well their advantages and disadvantages. According to their result, all the abovementioned N-RTK interpolation method can acceptably reduce the effect of the GPS distancedependent errors with almost similar performance. Another research had been conducted by Shariff et al., 2014, to evaluates these N-RTK interpolation method network coefficient value based on various user location, various master location and different number of reference station (three and four).

From the result, all above-mentioned N-RTK interpolation method performance is similar when the user near to the references or master station, however, the noise of correction term was different as the users located at various location especially outside of the network. 
Additionally, the user whose location almost at the center of the network will have proper weighting coefficient thus lead to better positioning performances.

Compared to all above-mentioned N-RTK interpolation method, the LIM and LCM had more advantages compared to others N-RTK interpolation method which has computed the lowest noise value when the users at the center or inside the network, effective when the user located near to the reference station especially master station, compatible when using three reference station and has insignificant improvement of the noise with adding fourth reference station. By comparing all the above-mentioned N-RTK interpolation method, the LIM has been chosen to generate the network coefficient value for specific user location. Figure 3 shows the distribution of reference stations and user location for network coefficient value computation purpose.

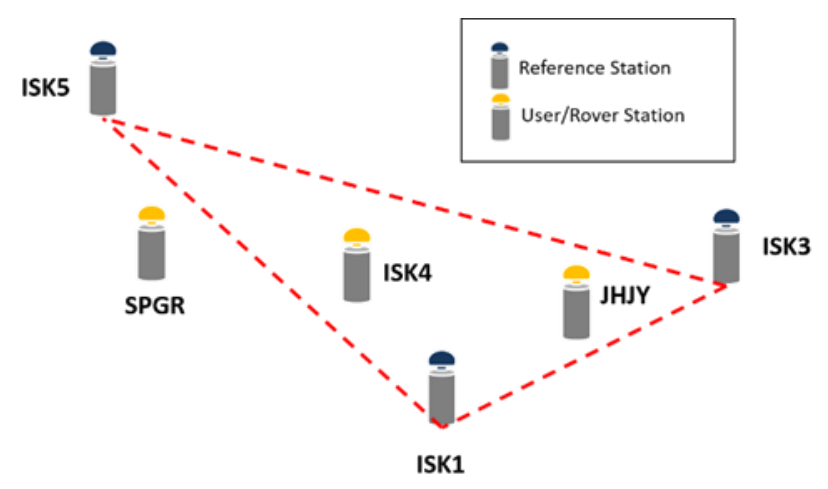

Figure 3: Distribution of User Location for Network Coefficient Computation Purpose.

Based on Figure 3, all the location are distributed based on real monument of reference station of ISKANDARnet and Malaysian Real-Time-Kinematic Network (MyRTKnet).

\section{Generation of GPS Dispersive and Non-Dispersive Network Correction}

The network correction can be delivered in two ways which are the separated GPS dispersive and non-dispersive network correction or the 'lump sum' network correction which combine both GPS dispersive and non-dispersive network correction (Musa, 2007). To attain better modelling of GPS distance-dependent errors, the separated GPS dispersive and non-dispersive network correction is proposed. This is because, when the GPS signal passing through the atmospheric layer, the signal becomes delays, the delay however was in different shape when it comes to each of the layer especially for ionospheric and tropospheric layer. The signal is accelerated when it comes to ionospheric layer while slowing down when it comes to tropospheric layer.

This approach however is to partition according to whether there are dispersive or nondispersive. This partition is done by GF and IF carrier-phased linear combination which has been discussed in Section 2.1 to obtain true GPS dispersive and non-dispersive network residual components. Next, the GF and IF network correction can be written as follow (Musa, 2007):

$$
\begin{gathered}
\text { Non_Disp_Corr }=\alpha_{1} \cdot V_{1,3}^{I F}+\alpha_{2} \cdot V_{2,3}^{I F} \\
\text { Disp_Corr }=\alpha_{1} \cdot V_{1,3}^{G F}+\alpha_{2} \cdot V_{2,3}^{G F}
\end{gathered}
$$


Where $\alpha_{1}$ and $\alpha_{2}$ is the network coefficient 1 and 2 respectively, $V_{1,3}^{I F}$ and $V_{2,3}^{I F}$ is the GPS nondispersive and dispersive residual components for reference station 1 and master station 3 . While $V_{2,3}^{I F}$ and $V_{2,3}^{G F}$ is the GPS non-dispersive and dispersive residual components for reference station 2 and master station 3 .

Other effect such as orbital errors also may exists in the network correction. One way to minimized this error is by utilizing the precise ephemeris such as final orbit, rapid orbit or ultrarapid orbit which are available via IGS product website (IGS, 2021). The reduction number of orbital errors increase starting from final orbit to ultra-rapid orbit. To avoid such significant number of orbital errors in network correction, the precise ephemeris namely ultra-rapid orbit is utilized. Another reason is to equate the same level of orbital errors with the ISKANDARnet N-RTK positioning system.

The result however does not have any effect on GPS dispersive network residual components since the orbital error have been eliminated during GF carrier-phased linear combination processing compared to GPS non-dispersive network residual components which are improved rather than by using broadcast ephemeris. The effect of orbital error can be seen by comparison of two result from IF carrier-phased linear combination by using precise and broadcast orbit according to (Musa, 2007).

\section{Results}

Figure 4 and Figure 5 shows the GPS dispersive network residual components by using GF carrier-phased linear combination while Figure 6 and Figure 7 shows the GPS non-dispersive network residual components by using IF carrier-phased linear combination.
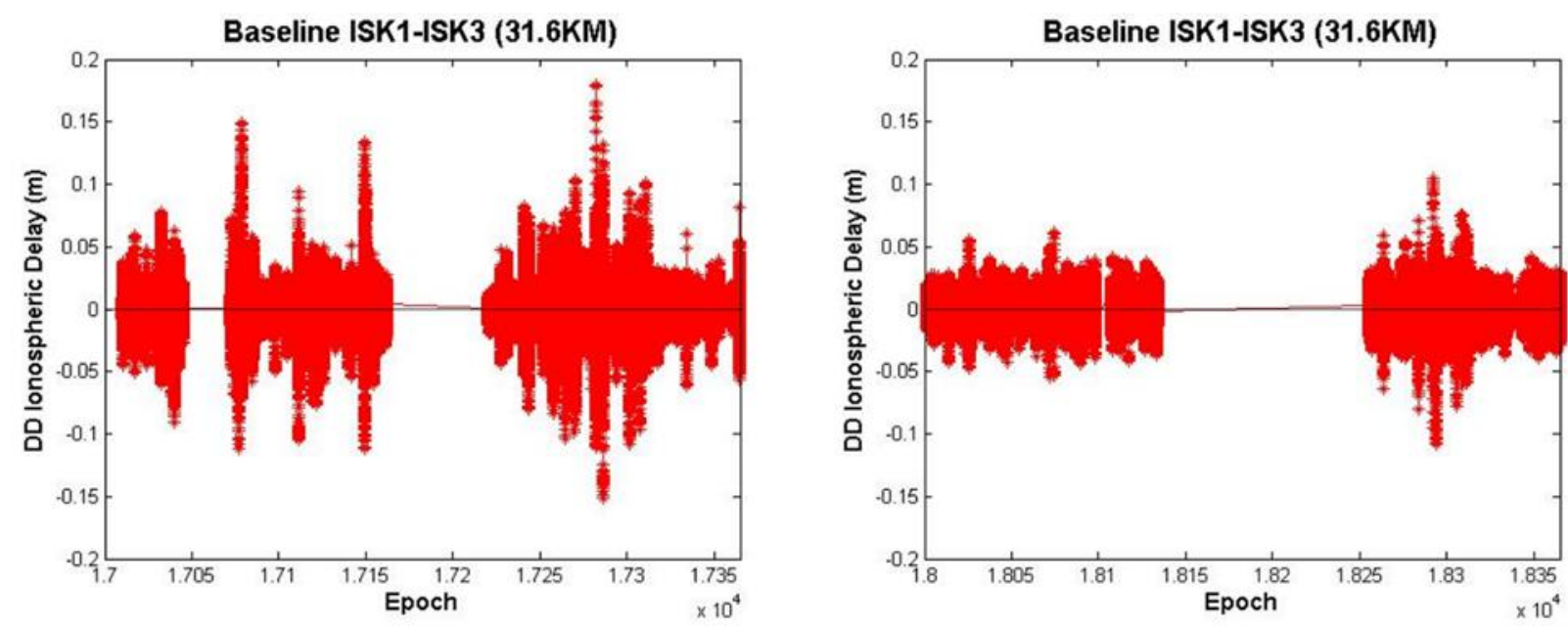

Figure 4: GPS Dispersive Residual Components for Baseline ISK1 to ISK3 Station. 

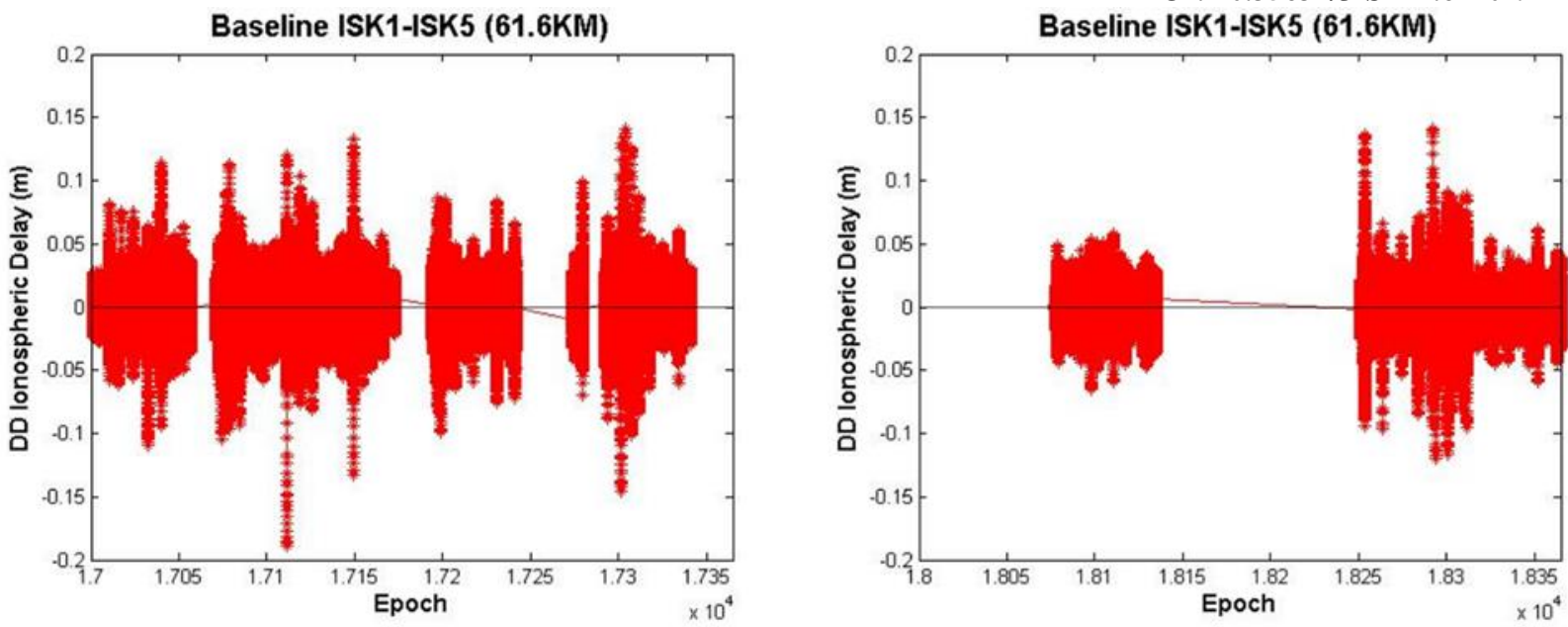

Figure 5: GPS Dispersive Residual Components for Baseline ISK1 to ISK5 Station.
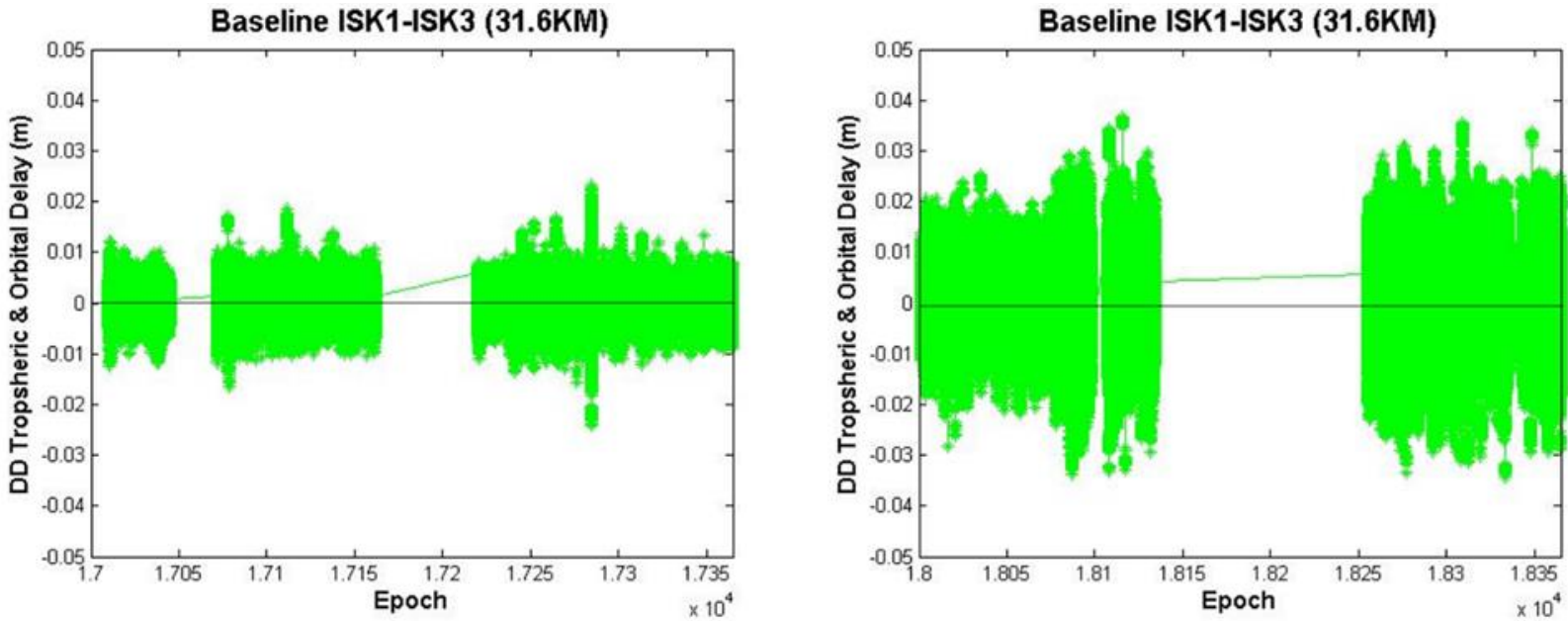

Figure 6: GPS Non-Dispersive Residual Components for Baseline ISK1 to ISK3 Station.
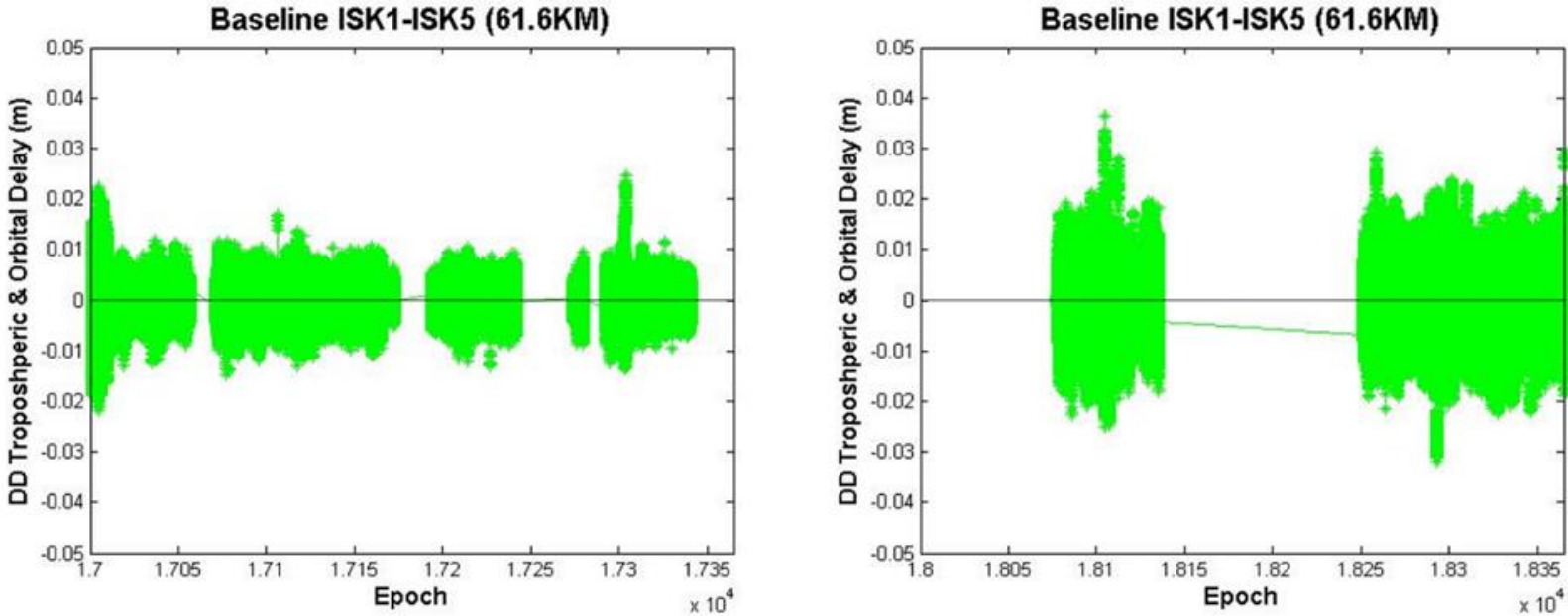

Figure 7: GPS Non-Dispersive Residual Components for Baseline ISK1 to ISK5 Station.

Figure 4 to Figure 7 shows the variation of GPS dispersive and non-dispersive network residual components for all satellite combination during 2 years of observation from day of year (DOY) 001 in year 2017 to DOY 365 in year 2018. The empty line or straight line shown in Figure 4 
until Figure 7 indicates that there is no residual data being estimated. One of the reasons is due to no data being observe among two stations of each baseline. Another reason is due to the automatic selection of baseline inside Bernese GNSS software namely 'OBS-MAX' which the selection was highly priority to the greatest number of common observation (correlation) for the associated station during baseline processing (Dach et al., 2015). Other reason is due to bad or damaged data observe generally because of GPS receiver or antenna hardware problem.

From the result, it can be seen that the GPS dispersive residual components indeed distance dependent while the GPS non-dispersive residual seems not. Further analysis on this GPS dispersive and non-dispersive residual components can be done through statistical analysis for validation purpose. Table 1 and Table 2 shows the average, standard deviation and variance of the estimated GPS dispersive and non-dispersive network residual components respectively to capture the mean value, the amount of dispersion and how spread out the residual is for each of these residual components.

Table 1: Statistical Analysis on GPS Dispersive Network Residual Components.

\begin{tabular}{c|c|c|c|c}
$\begin{array}{c}\text { GPS } \\
\text { Dispersive } \\
\text { Components }\end{array}$ & Baseline & Average (m) & $\begin{array}{c}\text { Standard } \\
\text { Deviation }(\mathrm{m})\end{array}$ & Variance (m) \\
\hline \multirow{2}{*}{ Year 2017 } & $\begin{array}{c}\text { ISK1 - ISK3 } \\
(31.6 \mathrm{~km})\end{array}$ & $-2.5908 \times 10^{-5}$ & 0.0040 & $1.6274 \times 10^{-5}$ \\
\cline { 2 - 5 } & $\begin{array}{c}\text { ISK1 - ISK5 } \\
(61.6 \mathrm{~km})\end{array}$ & $-3.0131 \times 10^{-5}$ & 0.0071 & $5.1100 \times 10^{-5}$ \\
\hline \multirow{2}{*}{ Year 2018 } & $\begin{array}{c}\text { ISK1 - ISK3 } \\
(31.6 \mathrm{~km})\end{array}$ & $1.4839 \times 10^{-5}$ & 0.0054 & $2.8907 \times 10^{-5}$ \\
\cline { 2 - 5 } & $\begin{array}{c}\text { ISK1 - ISK5 } \\
(61.6 \mathrm{~km})\end{array}$ & $-7.5554 \times 10^{-7}$ & 0.0061 & $3.7205 \times 10^{-5}$ \\
\hline
\end{tabular}

Table 2: Statistical Analysis on GPS Non-Dispersive Network Residual Components.

\begin{tabular}{c|c|c|c|c}
\hline $\begin{array}{c}\text { GPS Non- } \\
\text { Dispersive } \\
\text { Components }\end{array}$ & Baseline & Average (m) & $\begin{array}{c}\text { Standard } \\
\text { Deviation }(\mathrm{m})\end{array}$ & Variance (m) \\
\hline \multirow{2}{*}{ Year 2017 } & $\begin{array}{c}\text { ISK1 - ISK3 } \\
(31.6 \mathrm{~km})\end{array}$ & $-2.5442 \times 10^{-6}$ & 0.0015 & $2.1611 \times 10^{-6}$ \\
\cline { 2 - 5 } & $\begin{array}{c}\text { ISK1 - ISK5 } \\
(61.6 \mathrm{~km})\end{array}$ & $-1.0880 \times 10^{-5}$ & 0.0016 & $2.6507 \times 10^{-6}$ \\
\hline \multirow{2}{*}{ Year 2018 } & $\begin{array}{c}\text { ISK1 - ISK3 } \\
(31.6 \mathrm{~km})\end{array}$ & $-5.3195 \times 10^{-6}$ & 0.0043 & $1.8798 \times 10^{-5}$ \\
\cline { 2 - 5 } & $\begin{array}{c}\text { ISK1 - ISK5 } \\
(61.6 \mathrm{~km})\end{array}$ & $6.8071 \times 10^{-7}$ & 0.0031 & $9.8823 \times 10^{-6}$ \\
\hline
\end{tabular}

From Table 1 and Table 2, it can be seen that both GPS dispersive and non-dispersive indeed dependent on baseline length except for GPS non-dispersive residual components in year 2018 where the residual for baseline ISK1 to ISK3 is higher compared to ISK1 to ISK5. The most influential impact on non-dispersive component residual was known as the water vapor which varies depending on the temperature and pressure of the air compared to dry delay which it can be model through ideal gas law and hydrostatic model. 
The reason for the GPS non-dispersive residual components for year 2018 was not distancedependent maybe due to significant variation of water vapor content between ISK1 and ISK3 station compared to ISK1 to ISK5 during observation. Salihin et al., (2017) shows the time series analysis of ZPD and Integrated Water Vapor (IWV) and their characteristic on their seasonal variation during monsoon seasons.

An alternative way to identified this problem is by in-depth analysis on wet delays at each of the baseline. To limit the outcome of this paper, further analysis on wet delays is not included in this paper. As a conclusion, the distribution of GPS dispersive and non-dispersive residual components agreed depends on baseline length. However, there are insignificant differences between those two baselines GPS dispersive and non-dispersive residual since both baseline length can be considered as in the same group of baselines which is short baseline. Musa, 2007 shows the evaluation of GPS dispersive and non-dispersive residual components based on short, medium and long baseline length.

The core in N-RTK is to model these GPS distance-dependent errors either for lump or separated components. The network coefficient often calls as 'correction term' are one of the main parts to generate the network correction. This correction term utilizes the network residual vectors between master and reference station once the ambiguity has been resolved. Several commonly used N-RTK interpolation method had been discussed in Section 2.2. Most of these N-RTK interpolation method use n-1 independent error vector to model the GPS distancedependent errors at the specific user station (Shariff, 2011).

In this paper, the LIM are used as N-RTK interpolation method to model these GPS dispersive and non-dispersive network residuals. Table 3 shows the network coefficient value for users based on LIM N-RTK interpolation method.

Table 3: Network Coefficient Value Based on LIM for Specific User Random Location.

\begin{tabular}{|l|l|l|l|}
\hline LIM & $\begin{array}{l}\text { User1 } \\
\text { (ISK4) }\end{array}$ & $\begin{array}{l}\text { User2 } \\
\text { (JHJY) }\end{array}$ & $\begin{array}{l}\text { User3 } \\
\text { (SPGR) }\end{array}$ \\
\hline$\alpha 1$ & 0.1723 & 0.6243 & -0.6019 \\
\hline$\alpha 2$ & 0.2408 & 0.0376 & 0.4649 \\
\hline$\sum_{i=1}^{3} \alpha_{i}$ & 0.4131 & 0.6619 & -0.13701 \\
\hline$\sqrt{\sum_{i=1}^{3} \alpha_{i}^{2}}$ & 0.29612 & 0.6254 & 0.7605 \\
\hline
\end{tabular}

Table 3 shows that the network coefficient value of a user is higher when the user located near to reference station. For instance, User2 have the largest network coefficient value as indicates by $\alpha 1$ which are highly influenced by the reference station of ISK3. Even for the user outside of network such as User3, the network coefficient value also shows the highest and lowest indicates by $\alpha 1$ and $\alpha 2$ which are highly influenced by reference station of ISK 3 and ISK 5 
respectively. Interestingly, it was found that the network coefficient $\alpha 1$ and $\alpha 2$ has been properly distributed for the user located almost at the center of the network which is at User1.

The square sum of network coefficient value indicates the noise for the correction term which means the smaller the better according to Dai et al., 2003 and Shariff et al., 2014. Figure 8 shows the bar chart for the last row in Table 3 that representing the square sum of network coefficient value of LIM.

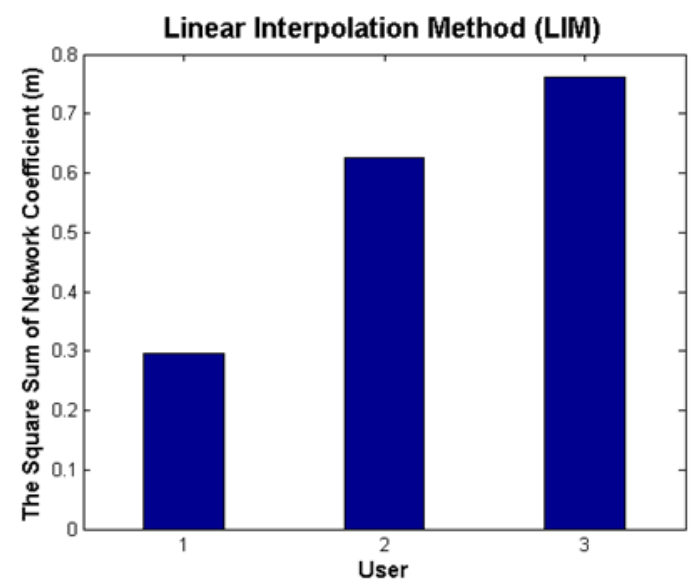

Figure 8: The Square Sum of Network Coefficient or Noise for the Correction Terms of LIM.

Figure 8 shows that the noise stated the highest when the user situated outside the network. Meanwhile, the lowest noise level can be seen when the user situated almost at the center of the network. It can be concluded that this result shows that the network coefficient based on LIM was indeed location-based dependency according to Shariff et al., 2014. Furthermore, the performance and characteristic of LIM in this paper is the same compared to as Dai et al., 2003 and Shariff, 2014. Thus, by locating user at the center of the network, the noise will be reduced by LIM N-RTK interpolation method.

The GPS dispersive and non-dispersive network correction can be generated by multiplying the network coefficient value and GPS dispersive and non-dispersive residual respectively. The network coefficient value will be act as a weighting for network correction dissemination. Figure 9 and Figure 10 shows the generated GPS dispersive and non-dispersive network correction for ISKANDARnet based on previous LIM network coefficient value and GPS dispersive and non-dispersive network residual components vector. 


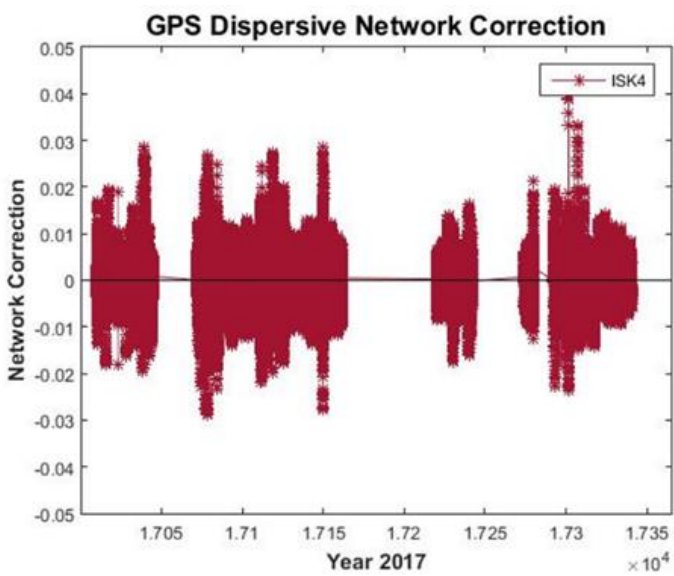

DOI: 10.35631/JISTM.624029

Figure 9: GPS Dispersive Network Correction Based on LIM Network Coefficient

Value and GPS Dispersive Residual Components Vector.
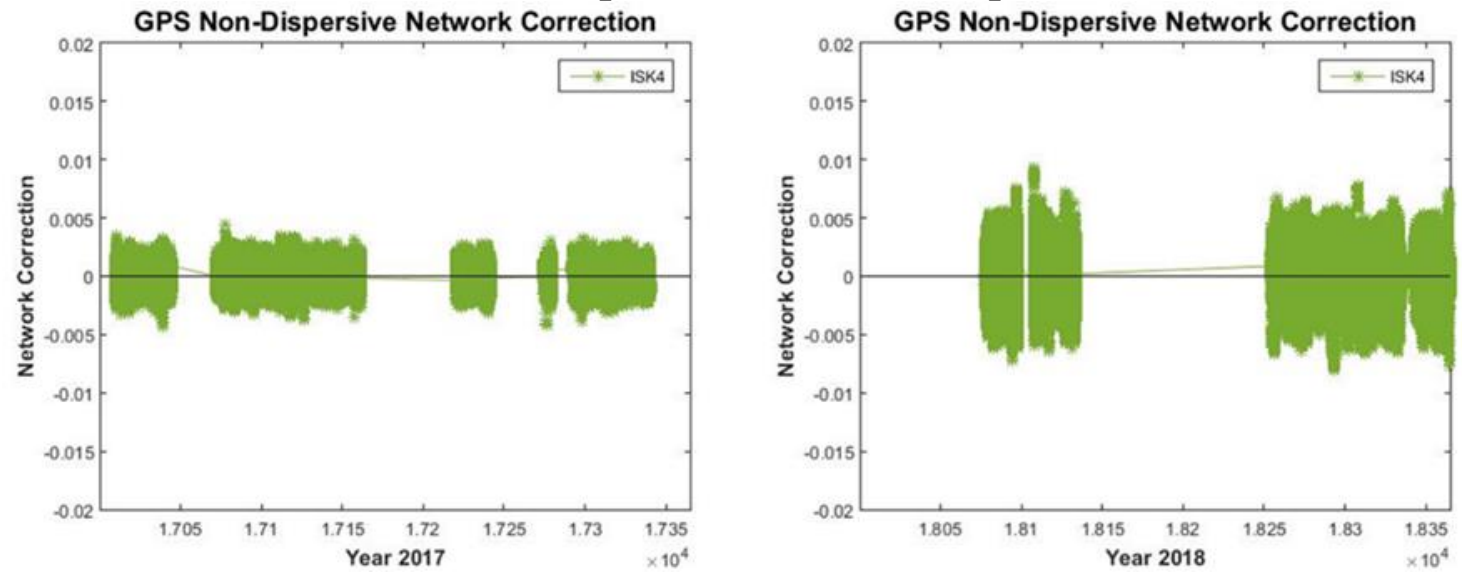

Figure 10: GPS Non-Dispersive Network Correction Based on LIM Network Coefficient Value and GPS Non-Dispersive Residual Components Vector.

Figure 9 and Figure 10 shows the trend of GPS dispersive and non-dispersive network correction based on GF and IF network correction algorithm. Noticed that only the GPS dispersive and non-dispersive residual components with the same pair of satellite for each baseline are used to generate both GPS dispersive and non-dispersive network correction. The empty line and straight line showed in Figure 9 and Figure 10 indicates there are no network correction exist for some pairs of satellites. The reason has been discussed above due to unavailability of GPS observation data, Bernese software processing technique and etc.

The GPS dispersive and non-dispersive network correction are generated based on estimated GPS dispersive and non-dispersive residual components respectively which has been weighted and modelled by LIM. From the result, it can be seen that the GPS dispersive network correction shows more active compared to GPS non-dispersive network correction. Wei et al., 2006 also mentioning that the ionospheric delay is more active compared to tropospheric delay. Further analysis to evaluates the trend analysis for GPS dispersive and non-dispersive can be done via statistical analysis. Table 4 shows the statistical analysis for GPS dispersive and nondispersive network correction. 
Table 4: Statistical Analysis on GPS Dispersive and Non-Dispersive Network Correction.

\begin{tabular}{c|c|c|c|c}
\hline $\begin{array}{c}\text { Network } \\
\text { Correction }\end{array}$ & Year & Average $(\mathrm{m})$ & $\begin{array}{c}\text { Standard } \\
\text { Deviation }(\mathrm{m})\end{array}$ & Variance $(\mathrm{m})$ \\
\hline \multirow{2}{*}{ Dispersive } & 2017 & $1.2665 \times 10^{-5}$ & 0.0016 & $2.6931 \times 10^{-6}$ \\
\cline { 2 - 5 } & 2018 & $-5.9761 \times 10^{-6}$ & 0.0018 & $3.1177 \times 10^{-6}$ \\
\hline $\begin{array}{c}\text { Non- } \\
\text { Dispersive }\end{array}$ & 2017 & $-3.7317 \times 10^{-6}$ & 0.0005 & $2.3232 \times 10^{-7}$ \\
\cline { 2 - 5 } & 2018 & $3.0195 \times 10^{-6}$ & 0.0012 & $1.3673 \times 10^{-6}$ \\
\hline
\end{tabular}

From Table 4, it can be seen that the mean value during these two years of observation for GPS dispersive and non-dispersive network correction are vice versa which are higher in year 2018 for GPS dispersive components while higher in year 2017 for GPS non-dispersive components. Meanwhile, the standard deviation shows that in year 2018, both GPS dispersive and nondispersive network correction are higher compared to in year 2017. However, there are no significant differences occur on GPS dispersive network correction during these two years of observation compared to GPS non-dispersive network correction. Overall analysis shows in year 2018, both GPS dispersive and non-dispersive network correction are more active compared in year 2017.

Unfortunately, this analysis however can't be confirmed yet since there are too many missing of GPS observation data during these two years of observation. In other words, there are high possibilities that the wholly observed GPS dispersive and non-dispersive network correction can significantly change the outcome of statistical analysis since the variation are completely unpredicted for both GPS dispersive and non-dispersive components.

An alternative way to improve this statistical analysis is by increase the amount of GPS observation data from two years to more years. Others alternative way is by changing the GPS observation period to another years of GPS observation period. To improve the strength and reliability of this statistical analysis, this paper is strongly recommended only to increase the amount of GPS observation data from two years to more. By increasing the amount of GPS observation data, the quality of this statistical analysis is expected to be improve.

The standard deviation value based on this statistical analysis can be used to support the determination and realization of threshold limit value for ISKANDARnet network correction integrity monitoring. By extending the trend analysis for both GPS dispersive and nondispersive network correction, the quality of threshold limit value in this case is expected to be significantly improve. Other integrity monitoring for network correction concept as well are being review to strengthen the development of integrity monitoring for network correction of ISKANDARnet N-RTK positioning system.

\section{Conclusion}

As a conclusion, the estimated GPS dispersive network residual shows it is depending on baseline length which is the longer the baseline the higher the residual components. However, the GPS non-dispersive network residual were not. The reason behind this phenomenon has been discussed in Section 3.0. An alternative way to verify this rare situation is by in-depth wet delay analysis on GPS non-dispersive network residuals. 


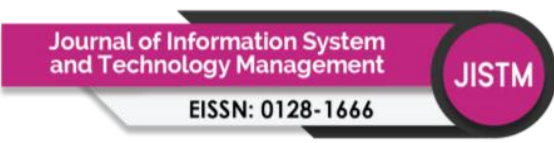

Volume 6 Issue 24 (December 2021) PP. 312-325

DOI: 10.35631/JISTM.624029

The performance of LIM N-RTK interpolation is highly dependent on master and reference station location to the user. The near the user to reference station, the higher the network coefficient value. The noise of correction however can be reduced by locating user at the center of the network. Meanwhile, the result for network correction shows that the GPS dispersive network correction are more active compared to GPS non-dispersive network correction.

Further analysis has been discussed that in year 2018, both GPS dispersive and non-dispersive network correction are more active compared to in year 2017. However, the average shows that both GPS dispersive and non-dispersive network correction are in different condition during these two years period. It can be concluded that this analysis required more additional GPS observation data as to improve the reliability and strength for the statistical analysis. The more the GPS data the more quality the statistical analysis.

\section{Acknowledgement}

The authors would like to acknowledge the Research University Grant (RUG) vot number R.J130000.7352.4J341 for providing funds in this project. Special thanks also to the Geomatic Innovation Research Group (GnG) and International GNSS Service (IGS) for providing ISKANDARnet and IGS CORS GPS data respectively.

\section{References}

Blewitt, G. (1989). Carrier phase ambiguity resolution for the Global Positioning System applied to geodetic baselines up to $2000 \mathrm{~km}$. Journal of Geophysical Research: Solid Earth, 94(B8), 10187-10203.

Bosy, J. (2005). Data processing of local gps network located in a mountain area. Acta Geodynamica et Geromaterialia, 2(3), 49-57.

Carrano, C. S., Groves, K. M., McNeil, W. J., \& Doherty, P. H. (2013, January). Direct measurement of the residual in the ionosphere-free linear combination during scintillation. In Proceedings of the 2013 international technical meeting of the Institute of Navigation (pp. 585-596).

Dach, R., Lutz, S., Walser, P., \& Fridez, P. (2015). Bernese GNSS software version 5.2.

Dai, L., Han, S., Wang, J., \& Rizos, C. (2003). Comparison of interpolation algorithms in network-based GPS techniques. NAVIGATION, Journal of the Institute of Navigation, 50(4), 277-293.

Krypiak-Gregorczyk, A., \& Wielgosz, P. (2018). Carrier phase bias estimation of geometryfree linear combination of GNSS signals for ionospheric TEC modeling. GPS Solutions, 22(2), 1-9.

IGS (2021) International GNSS Service products, http://www.igs.org/products (accessed July 2021).

Leick, A., Rapoport, L., \& Tatarnikov, D. (2015). GPS satellite surveying. John Wiley \& Sons.

Lim, S., Heo, Y., \& Rizos, C. (2008). A web-based real-time monitoring system for GNSS data quality and integrity. FIG Working Week 2008.

Musa, T. A. (2007). Analysis of residual atmospheric delay in the low latitude regions using network-based GPS positioning (Doctoral dissertation, University of New South Wales, Sydney, Australia).

Nykiel, G., \& Figurski, M. (2016, January). Precise point positioning method based on widelane and narrow-lane phase observations and between satellites single differencing. In Proceedings of the 2016 International Technical Meeting of The Institute of Navigation (pp. 1055-1066). 
Rubinov, E., Wonnacott, R., Fuller, S., \& Collier, P. (2011). Integrity monitoring of CORS Networks-TrigNet case study. South African Journal of Geomatics, 1(1), 1-13.

SALIHIN, S., MUSA, T. A., \& RADZI, Z. M. (2017). SPATIO-TEMPORAL ESTIMATION OF INTEGRATED WATER VAPOUR OVER THE MALAYSIAN PENINSULA DURING MONSOON SEASON. International Archives of the Photogrammetry, Remote Sensing \& Spatial Information Sciences, 42.

Shariff, N. S. M., Musa, T. A., Ses, S., Omar, K., Rizos, C., \& Lim, S. (2009, August). ISKANDARnet: a network-based real-time kinematic positioning system in ISKANDAR Malaysia for research platform. In South East Asian survey congress, Bali international convention center, Nusa Dua, Bali, Indonesia (pp. 4-7).

Shariff, N. S. (2011). A Network-Based Real-Time Kinematic Positioning System. Skudai, Johor: Universiti Teknologi Malaysia (UTM).

Shariff, N. S., Musa, T. A., \& Othman, R. (2014). Evaluation of N-RTK interpolation with location-based dependency. Jurnal Teknologi, 71(4).

Wang, J., Feng, Y., \& Wang, C. (2009, September). Rover autonomous integrity monitoring of GNSS RTK positioning solutions with multi-constellations. In Proceedings of the 22nd International Technical Meeting of the Satellite Division of The Institute of Navigation (ION GNSS 2009) (pp. 1361-1370).

Wei, E., Chai, H., An, Z., \& Liu, J. (2006). VRS virtual observations generation algorithm. Journal of Global Positioning Systems, 5(1-2), 76-81.

Wielgosz, P. (2011). Quality assessment of GPS rapid static positioning with weighted ionospheric parameters in generalized least squares. GPS solutions, 15(2), 89-99.

Zhang, X., Cai, W., Wang, B., \& Hou, Y. (2019). The advantages of the original combination over narrow lane combinations in multi-frequency GNSS orientating. Journal of Computational Methods in Sciences and Engineering, 19(3), 681-694. 